Jeana Cost, LPC, CEDS

ACUTE at Denver Health, Denver, CO

Eating Recovery Center, Denver, CO
Mori J. Krantz, MD

Department of Medicine, University of Colorado School of Medicine, Aurora, CO
Philip S. Mehler, MD, FACP, FAED

Founder, ACUTE at Denver Health, Denver, CO;

Eating Recovery Center, Denver, CO; Professor,

Department of Medicine, University of Colorado

School of Medicine, Aurora, CO

\title{
Medical complications of anorexia nervosa
}

\section{ABSTRACT}

Anorexia nervosa is a mental illness characterized by selfstarvation, marked weight loss, and malnutrition. As the illness worsens, numerous medical complications develop throughout the body. Some of these resolve with effective nutritional rehabilitation and weight gain, whereas others can lead to permanent damage.

\section{KEY POINTS}

The structural cardiac hallmark of this disease is myocardial atrophy characterized by a reduction in left ventricular mass index and volume, which commonly results in mitral valve prolapse.

Most female patients are amenorrheic and have low estrogen levels because they have reverted to a prepubertal state; male patients have low testosterone levels.

Marked loss of bone mineral density occurs, which can lead to early osteopenia and osteoporosis, even in adolescent patients, and this loss may be permanent.

Pulmonary complications include spontaneous pneumothorax, pneumomediastinum, and aspiration pneumonia.

Patients may also have generalized brain atrophy, damaged gray and white matter, and cognitive deficits that persist after treatment.
A norexia nervosa (AN) is a common A mental illness characterized by self-starvation, excessive weight loss, and malnutrition. Unlike in most other mental health disorders, in which physical health may be completely normal, compromised physical health is inextricably connected with this illness. Multiple concomitant medical complications occur throughout the body and become more pronounced as the severity of the illness increases. This review discusses these complications, many of which resolve with effective nutritional therapy and weight gain. Others can lead to permanent damage.

\section{INCIDENCE AND ETIOLOGY}

The peak age of onset is during adolescence. Incidence rates are increasing in both males and females, although the disease primarily affects adolescent girls and young women. Although estimates vary, $1 \%$ or more of women may develop AN during their lifetime, and the average duration of the illness is 6 years. ${ }^{1,2}$

AN is the deadliest of all mental illnesses. Mortality rates are as high as $5 \%,{ }^{2}$ and patients carry a 10 -fold increased risk for suicide. ${ }^{2}$ About 20\% of deaths in patients with AN are the result of suicide. ${ }^{3}$

The etiology of AN is complex, with many genetic, psychological, environmental, and social variables at play. Patients who have a first-degree relative with $\mathrm{AN}$, for example, have a 10-fold greater risk of having the illness themselves. ${ }^{4}$ Those with comorbid psychological illnesses such as depression, anxiety, and substance abuse are also at increased risk. A number of factors can trigger or exacerbate this eating disorder and sustain it, including societal and media pressure to appear thin, 
diet culture, occupations that require a lean physique (eg, sports, modeling), lack of a support system, and traumatic events (eg, sexual assault, physical abuse, neglect).

\section{EVALUATION}

The assessment and diagnosis of AN was updated in the 5th edition of the Diagnostic and Statistical Manual of Mental Disorders (DSM5). ${ }^{5}$ Hallmarks of the illness include intentional caloric restriction resulting in weight loss, intense fear of gaining weight, and body image distortions (ie, believing that they are grotesquely fat, when in fact they are normal or even underweight).

The DSM-5 includes a severity index for evaluating body mass index (BMI), which allows healthcare providers to assess the severity of malnutrition and the proper level of necessary care. The index has 4 categories: mild $\left(\right.$ BMI $\left.>17 \mathrm{~kg} / \mathrm{m}^{2}\right)$, moderate (16 to 16.99$)$, severe (15 to 15.99$)$, and extreme $(<15)$.

The DSM-5 also allows professionals to assess for one of two subtypes of the illness. The restricting type is associated with the previously mentioned symptoms and behaviors and does

Sinus bradycardia, profound reversible sinus node dysfunction, and orthostatic hypotension are uniformly observed not include regular bingeing of food, whereas the binge-eating/purging type is marked by bingeing and purging behaviors such as selfinduced vomiting or the misuse of diuretics or laxatives, or both. The difference between the latter subtype and bulimia nervosa is that bulimia does not include any weight-loss criteria, and the behaviors are accompanied by a sense of lack of control.

\section{MEDICAL AND THERAPEUTIC APPROACHES}

Treatment options are subject to ongoing debate and a lack of empiric evidence. The patient's medical stability, psychiatric stability, AN severity, age, support system, and duration of illness must be assessed. In the United States, patients have access to levels of care on the spectrum from general outpatient care to acute medical inpatient hospitalization. Therapeutic approaches vary based on age and level of care.

While the most commonly used approach for children and adolescents is family-based therapy, a much wider variety of treatments can be used in adults, such as cognitive reme- diation therapy, exposure therapy, dialectical behavior therapy, and acceptance and commitment therapy. That no single approach has emerged as the definitive evidence-based optimal treatment further suggests that there are many other factors to consider. ${ }^{6}$

\section{PROGNOSIS}

Recovery rates for AN vary considerably. Studies that focused on adolescents report recovery rates from $17.2 \%$ to $50 \%$, and those that focused on adults report recovery rates from $13 \%$ to $42.9 \%{ }^{1}$

Studies show that while eating disorders themselves are undertreated, a large number of patients receive only partial treatment for other comorbid issues such as depression, anxiety, substance abuse, and medical concerns. Improved recognition and assessment of eating disorder symptoms will lead to effective intervention and treatment and thus benefit the patient. ${ }^{\text {? }}$

The rest of this review discusses the medical complications associated with AN.

\section{CARDIAC PROBLEMS}

Changes in the structure and function of the heart, autonomic parameters, and cardiac repolarization have been noted in contemporary systematic reviews of AN. ${ }^{8}$

Myocardial atrophy, the structural hallmark of this disease, is characterized by a reduction in left ventricular mass index and an attendant decrease in left ventricular volume.

Mitral valve prolapse is common in AN. Although its mechanism has not been fully elucidated, it is thought to be a consequence of myocardial atrophy and reduced left ventricular chamber size leading to relative valvular laxity even in the absence of myxomatous valve degeneration. This "valvular-ventricular disproportion theory" suggests that either excessive mitral valve tissue or inadequate left ventricular cavity size results in prolapse. Supporting this theory is the observation that prolapse disappears in patients after weight restoration but recurs when patients lose weight again. ${ }^{9}$

In a cohort study, ${ }^{10}$ the authors observed mitral valve prolapse in most of their patients with severe AN but found no significant cor- 
relation between left ventricular dimension and prolapse. In contrast, a low heart rate had a significant correlation with mitral valve prolapse. Therefore, the cause of prolapse is likely multifactorial and may also be mediated by increased underlying vagal tone and resultant bradycardia.

Pericardial effusion may develop with progressive weight loss but generally remits with weight restoration and concurrent normalization of serum triiodothyronine $\left(\mathrm{T}_{3}\right)$ levels. ${ }^{11}$

Sinus bradycardia, profound reversible sinus node dysfunction, and orthostatic hypotension are uniformly observed in patients with severe AN. ${ }^{12}$ Electrocardiography may be appropriate depending on the patient's body mass index (BMI). It is unlikely to reveal much if the BMI is higher than $17 \mathrm{~kg} / \mathrm{m}^{2}$, but is likely to show significant bradycardia or other arrhythmia if less than 15. As BMI drops, bradycardia and hypotension become more pronounced.

There are no functional cardiovascular hallmarks diagnostic of AN. However, tissue Doppler indices of diastolic dysfunction (impaired relaxation, ventricular stiffness) are present. ${ }^{13}$ Analogously, increased arterial pulse wave velocity, a measure of aortic stiffness, has also been described. ${ }^{14}$

Although studies show that patients with AN have lower exercise capacity, blood pressure, and peak cardiovascular indices (eg, oxygen consumption), actual systolic ventricular function is maintained at peak exercise. ${ }^{15}$ This preserved ejection fraction suggests that despite marked caloric restriction, deconditioning, and skeletal muscle atrophy, left ventricular systolic function remains ostensibly preserved.

Sudden cardiac death is often the cause of premature death in patients with AN. Autonomic dysfunction, as measured by reduced heart rate variability, has been described in AN patients, although a consistent pattern has not emerged when evaluated systematically. ${ }^{16,17}$ Similarly, delayed repolarization manifested as prolongation of the rate-corrected QT (QTC) interval on 12-lead electrocardiography has been posited as a likely cause. However, this association has been disproved by the single largest electrocardiographic study of AN patients, in which the population mean QTc interval was 417 ms. ${ }^{18}$
Although QTc prolongation and torsade de pointes occur in patients with eating disorders, the data linking QTc prolongation, AN, and risk of sudden death are confounded by the presence of concurrent hypokalemia and medications that block the delayed rectifier potassium ion channel.

Perhaps the most compelling piece of recent research that supports this interpretation is a 10-year population-based cohort study of 430 women with $\mathrm{AN}$ and 123 controls in Denmark. ${ }^{17}$ Overall, there was no difference in mean QTc interval or risk of prolonged QTc between patients with AN and healthy controls. However, patients with AN had a notably higher risk of a cardiac event (ventricular tachycardia, aborted cardiac arrest, or cardiac arrest) compared with controls (hazard ratio $10.4,95 \%$ confidence interval 2.6-41.6, $P=$ .001 ), as well as all-cause mortality (hazard ratio $11.2,95 \%$ confidence interval 5.1-24.5, $P$ $<.001)$. This relationship with cardiac events and all-cause mortality was not related to the baseline QTc interval. ${ }^{17}$

Decreased R-wave amplitude on electrocardiography is also commonly noted, though a relationship with major adverse cardiac events has not been demonstrated.

Despite the aforementioned cardiovascular complications observed in AN, an exact mechanism underlying the increased cardiovascular mortality risk in this disorder has not been firmly established. One possibility is subclinical left ventricular systolic dysfunction as manifested by abnormalities in myocardial torsion and global longitudinal systolic strain. ${ }^{19,20}$ Another possibility is focal regional fibrosis as a nidus for malignant ventricular arrhythmia, which has been suggested by late gadolinium enhancement on magnetic resonance imaging. ${ }^{21}$

We believe further investigation of subclinical cardiovascular dysfunction and long-term arrhythmia monitoring and larger populationbased cohort studies are needed to address the ongoing inordinately high risk of sudden cardiac death in this generally young population.

\section{GASTROINTESTINAL PROBLEMS}

As a direct result of weight loss and malnutrition, gastrointestinal transit time slows.
Gastroparesis and constipation

occur, especially as weight loss worsens 
Gastroparesis and constipation are therefore common in patients with AN, especially as weight loss becomes more severe. ${ }^{22}$ If the patient is symptomatic, metoclopramide or a macrolide antibiotic can be prescribed for a short time until some weight gain occurs.

Superior mesenteric artery syndrome occurs in patients with AN as a result of weight loss-induced atrophy of the mesenteric fat pad. Normally, the fat pad tethers the artery and prevents its medial movement, which could compress the third portion of the duodenum as it passes between the superior mesenteric artery and the aorta. Patients with superior mesenteric artery syndrome complain of fullness, nausea, and epigastric pain that begins soon after eating and is relieved by vomiting. The diagnosis is made by an upper gastrointestinal series or abdominal computed tomography. ${ }^{23}$ Superior mesenteric artery syndrome is treated with a soft or liquid diet until sufficient weight gain occurs to reconstitute the fat pad.

Diarrhea can occur early in the refeeding process due to small-bowel atrophy and a reduction in the absorptive area. A low level of blood diamine oxidase supports this etiology. ${ }^{24}$

The lungs are not immune to the ravages of anorexia nervosa
Liver disease. Aminotransferase levels are often elevated in AN. There are two main causes. Early on, before refeeding starts, it is likely caused by apoptosis-programmed hepatocyte cell death triggered by starvation. ${ }^{25}$ However, if levels start to abnormally elevate with refeeding, it is more likely to be caused by steatohepatitis, which responds to an alteration in the macro-composition of the diet with a reduction in calories from carbohydrates. ${ }^{26}$ Surprisingly, albumin levels are normal even with severe AN.

Functional bowel disorders are common in patients with $\mathrm{AN} .{ }^{27}$

\section{PULMONARY PROBLEMS}

For many years, the lungs were thought to be immune to the ravages of AN. However, we now know that this is not the case. ${ }^{28}$

Spontaneous pneumothorax and pneumomediastinum occur in patients with AN.

Aspiration pneumonia can occur with marked weight loss due to weakening of the pharyngeal muscles and swallowing problems, which can be identified by modified barium swallow studies.

Pulmonary function tests may be abnormal and show an obstructive pattern, but the cause is unknown.

\section{LOW WHITE BLOOD CELL, RED BLOOD CELL, PLATELET COUNTS}

Gelatinous marrow transformation occurs as malnutrition worsens. Specifically, serous fat atrophies in the bone marrow, and normal marrow fat is replaced by a thick mucopolysaccharide substance that impedes the egress of precursor cells from the bone marrow. ${ }^{29,30}$ This leads to trilinear hypoplasia with leukopenia, anemia, and thrombocytopenia detected in that order of decreasing frequency. ${ }^{31}$

Leukopenia. Interestingly, despite frank neutropenia, patients with AN are not at increased risk of infection, and thus neutropenic precautions are not needed. Similarly, the use of expensive growth factors is not indicated because the marrow reconstitutes quickly with nutritional rehabilitation.

Anemia in AN is typically normocytic, but when the red blood cell indices are abnormal, it is typically macrocytic, although vitamin $B_{12}$ and folate levels are not low..$^{32}$ Microcytic anemia is rare and requires additional evaluation.

\section{MULTIPLE ENDOCRINE ABNORMALITIES}

Many endocrine abnormalities occur in patients with AN.

Amenorrhea is present in most females, who have low estrogen levels due to reversion to a prepubertal state in the hypothalamicpituitary axis, ${ }^{33}$ and most males have low testosterone. Menses generally resume when approximately $95 \%$ of ideal body weight is achieved, ${ }^{34}$ although it can take 6 to 9 months for this to occur. Of note, pregnancy can occur even with amenorrhea and is dangerous for the patient and the fetus.

Low leptin levels normalize with weight restoration and nutritional rehabilitation. ${ }^{35}$ Leptin levels may correlate with onset of regular menses.

Growth hormone resistance accompanies AN, as do elevated serum cortisol levels. Most patients have euthyroid sick syndrome, which is self-limited and reverses after nutritional rehabilitation.

Hypoglycemia is most often detected in 
patients with more severe forms of the illness and BMIs of less than $15 \mathrm{~kg} / \mathrm{m}^{2}$. Hypoglycemia is a bad prognostic sign, as it portends hepatic failure and an inability to actualize gluconeogenesis and glycogenolysis. ${ }^{36}$

\section{LOSS OF MUSCLE AND BONE}

Sarcopenia. Even though patients with AN tend to be young, they have significant sarcopenia and loss of skeletal muscle mass. This in turn causes a dangerous state of weakness and increases the risk of falls in more severely ill patients. These complications are completely reversible with weight gain and physical therapy.

Bone loss. A serious and possibly irreversible complication of AN that correlates with the presence of sarcopenia is the loss of bone mineral density and a proclivity toward early development of osteopenia and osteoporosis, even in adolescent patients.

The etiology of this exuberant loss of bone mineral density is likely multifactorial and includes elevated cortisol levels, low leptin and sex hormone levels, low body weight, and growth hormone resistance. ${ }^{37}$ As a result, these patients are often left with a markedly increased risk of fragility fractures, even long after their AN has remitted. ${ }^{38}$

Loss of bone mineral density in patients with AN is different from that in postmenopausal women. In $A N$, it is not only due to increased resorption but also decreased bone formation. This "uncoupled" state is why the loss of bone mineral density is so marked in AN. ${ }^{37}$

Measuring bone mineral density is very important if the patient has had anorexia nervosa for more than 1 year or amenorrhea for more than 9 or 12 months because there is exuberant and severe loss of bone mineral density. On the other hand, urinary telopeptide levels are not indicated, as one can make the decision to treat osteoporosis on the basis of the DXA results.

Treatment of osteoporosis in AN is controversial. Most agree that osteopenia should be treated by weight restoration and resumption of menses along with adequate intake of calci- um and vitamin D. Some experts in the field, however, are more cautiously aggressive with the osteoporosis of AN and have advocated for judicious consideration of medical treatment with bisphosphonates, transdermal estrogen, denosumab, or teriparatide. Currently, the use of denosumab has been described only in case reports. Also, the adverse effects of each of these drug classes, although rare, need to be fully explained to the patient before proceeding to prescribe.

For many years, oral contraceptives were not recommended as a treatment for bone loss due to low efficacy reported in studies in patients with AN. However, a recent cross-sectional study suggests that oral contraceptives may have a very limited role in severe AN. ${ }^{39}$

Bone densitometry should be done every 2 years during the active phases of AN.

\section{NEUROLOGIC SYSTEM: BRAIN ATROPHY}

Anorexia nervosa is characterized by marked brain atrophy on brain imaging studies. Particular areas of the brain seem to be preferentially damaged, including both gray and white matter and areas of the insula and thalamus. ${ }^{40}$ With weight restoration, these brain-size abnormalities seem to reverse, but there may be ongoing cognitive deficits that persist as a secondary medical complication of AN with permanent adverse sequelae. Brain atrophy may explain the abnormalities in taste, smell, thalamic function, and temperature regulation as well as the overall mental slowness seen in persons with more severe forms of the illness.

\section{DERMATOLOGIC COMPLICATIONS}

Patients with AN can develop a variety of skin conditions, including xerosis, which results in painful, dry, and fissured skin, acrocyanosis, and lanugo hair growth on the sides of the face and along the spine. The hair growth occurs as a result of the body attempting to conserve heat and is not a sign of masculinization. ${ }^{41} \mathrm{Also}$ seen are brittle hair and nails and unexplained hypercarotenemia, which gives the skin a yellowish appearance.

\section{A recent cross-sectional study suggested that oral contraceptives may have a very limited role in the treatment of AN-induced osteoporosis}




\section{ANOREXIA NERVOSA}

\section{REFERENCES}

1. Hudson JI, Hiripi E, Pope HG Jr, Kessler RC. The prevalence and correlates of eating disorders in the National Comorbidity Survey Replication. Biol Psychiatry 2007; 61(3):348-358. doi:10.1016/j.biopsych.2006.03.040

2. Berends T, Boonstra N, van Elburg A. Relapse in anorexia nervosa: a systematic review and meta-analysis. Curr Opin Psychiatry 2018; 31(6):445455. doi:10.1097/YCO.0000000000000453

3. Arcelus J, Mitchell AJ, Wales J, Nielsen S. Mortality rates in patients with anorexia nervosa and other eating disorders: a meta-analysis of 36 studies. Arch Gen Psychiatry 2011; 68(7):724-731. doi:10.1001/archgenpsychiatry.2011.74

4. Himmerich H, Bentley J, Kan C, Treasure J. Genetic risk factors for eating disorders: an update and insights into pathophysiology. Ther Adv Psychopharmacol 2019; 9:2045125318814734. doi:10.1177/2045125318814734

5. American Psychiatric Association. Diagnostic and Statistical Manual of Mental Disorders, 5th ed. Arlington, VA: American Psychiatric Publishing 2013.

6. Murray SB, Quintana DS, Loeb KL, Griffiths S, Le Grange D. Treatment outcomes for anorexia nervosa: a systematic review and meta-analysis of randomized controlled trials. Psychol Med 2019; 49(4):535-544. doi:10.1017/S0033291718002088

7. Brockmeyer T, Friederich HC, Schmidt U. Advances in the treatment of anorexia nervosa: a review of established and emerging interventions. Psychol Med 2018; 48(8):1228-1256. doi: $10.1017 /$ S0033291717002604

8. Giovinazzo S, Sukkar SG, Rosa GM, et al. Anorexia nervosa and heart disease: a systematic review. Eat Weight Disord 2019; 24(2):199-207. doi:10.1007/s40519-018-0567-1

9. Cheng TO. Anorexia nervosa. Postgrad Med 1987; 82(5):32,35. pmid:3671202

10. Oka Y, Ito T, Matsumoto S, Suematsu H, Ogata E. Mitral valve prolapse in patients with anorexia nervosa: two-dimensional echocardiographic study. Jpn Heart J 1987; 28(6):873-882. doi:10.1536/ihj.28.873

11. Sachs KV, Harnke B, Mehler PS, Krantz MJ. Cardiovascular complications of anorexia nervosa: a systematic review. Int J Eat Disord 2016; 49(3):238-248. doi:10.1002/eat.22481

12. Cotter R, Lyden J, Mehler PS, Miceli J, Schuller J, Krantz MJ. A case series of profound bradycardia in patients with severe anorexia nervosa: thou shall not pace? HeartRhythm Case Reports 2019; 5(11): 511-515.

13. Escudero CA, Potts JE, Lam PY, De Souza AM, Mugford GJ, Sandor GG. An echocardiographic study of left ventricular size and cardiac function in adolescent females with anorexia nervosa. Eur Eat Disord Rev 2016; 24(1):26-33. doi:10.1002/erv.2409

14. Escudero CA, Potts JE, Lam PY, De Souza AM, Mugford GJ, Sandor GG Doppler echocardiography assessment of aortic stiffness in female adolescents with anorexia nervosa. J Am Soc Echocardiogr 2018; 31(7):784790. doi:10.1016/j.echo.2018.01.003

15. Escudero CA, Potts JE, Lam PY, et al. Echocardiographic assessment of ventricular function during exercise in adolescent female patients with anorexia nervosa. J Am Soc Echocardiogr 2019; 32(3):394-403.e3. doi:10.1016/j.echo.2018.10.014

16. Krantz MJ, Sabel AL, Sagar U, et al. Factors influencing QT prolongation in patients hospitalized with severe anorexia nervosa. Gen Hosp Psychiatry 2012; 34(2):173-177. doi:10.1016/j.genhosppsych.2011.08.003

17. Frederiksen TC, Krogh Christiansen M, Charmoth Østergaard P, et al. QTC interval and risk of cardiac events in adults with anorexia nervosa: a long term follow-up study. Circ Arrhythm Electrophysiol 2018; 11(8):e005995. doi:10.1161/CIRCEP.117.005995

18. Krantz MJ, Blalock DV, Tanganyika K, Farasat M, McBride J, Mehler PS. Is QTc-interval prolongation an inherent feature of eating disorders? A cohort study. Am J Med Published online March 9, 2020. doi:10.1016/j.amjmed.2020.02.015

19. Almeida-Jones ME, Suntharos $P$, Rosen $L$, et al. Myocardial strain and torsion quantification in patients with restrictive-type anorexia nervosa. J Am Soc Echocardiogr 2014; 27:B41-B42.

20. Morris R, Prasad A, Asaro J, et al. Markers of cardiovascular dysfunction in adolescents with anorexia nervosa. Glob Pediatr Health 2017;4
.2333794X17727423 doi:10.1177/2333794X17727423

21. Oflaz S, Yucel B, Oz F, et al. Assessment of myocardial damage by cardiac MRI in patients with anorexia nervosa. Int J Eat Disord 2013; 46(8):862866. doi:10.1002/eat.22170

22. Kamal N, Chami T, Andersen A, Rosell FA, Schuster MM, Whitehead WE. Delayed gastrointestinal transit times in anorexia nervosa and bulimia nervosa. Gastroenterology 1991; 101(5):1320-1324. doi:10.1016/0016-5085(91)90083-w

23. Mascolo M, Dee E, Townsend R, Brinton JT, Mehler PS. Severe gastric dilatation due to superior mesenteric artery syndrome in anorexia nervosa. Int J Eat Disord 2015; 48(5):532-534. doi:10.1002/eat.22385

24. Takimoto Y, Yoshiuchi K, Shimodaira S, Akabayashi A. Diamine oxidase activity levels in anorexia nervosa. Int J Eat Disord 2014; 47(2):203-205. doi:10.1002/eat.22202

25. Rautou PE, Cazals-Hatem D, Moreau R, et al. Acute liver cell damage in patients with anorexia nervosa: a possible role of starvation-induced hepatocyte autophagy. Gastroenterology 2008; 135(3):840-848.e1-e3. doi:10.1053/j.gastro.2008.05.055

26. Rosen E, Sabel AL, Brinton JT, Catanach B, Gaudiani JL, Mehler PS. Liver dysfunction in patients with severe anorexia nervosa. Int J Eat Disord 2016; 49(2):151-158. doi:10.1002/eat.22436

27. Wang X, Luscombe GM, Boyd C, Kellow J, Abraham S. Functional gastrointestinal disorders in eating disorder patients: altered distribution and predictors using ROME III compared to ROME II criteria. World J Gastroenterol 2014; 20(43):16293-16299. doi:10.3748/wjg.v20.i43.16293

28. Jensen VM, Stoving RK, Andersen PE. Anorexia nervosa with massive pulmonary air leak and extraordinary propagation. Int J Eat Disord 2017; 50(4):451-453. doi:10.1002/eat.22674

29. Abella E, Felia E, Granda I, et al. Bone marrow changes in anorexia nervosa are correlated with the amount of weight loss and not with other clinical findings. Am J Clin Pathol 2002; 118(4):582-588. doi:10.1309/2Y7X-YDXK-006B-XLT2

30. Eckland K, Vajapeyam S, Mulkern RV, et al. Bone marrow fat content in 70 adolescent girls with anorexia nervosa: magnetic resonance imaging and magnetic resonance spectroscopy assessment. Pediatr Radiol 2017; 47(8):952-962. doi:10.1007/s00247-017-3856-3

31. Hutter G, Ganepola S, Hofmann WK. The hematology of anorexia nervosa. Int J Eat Disord 2009; 42(4):293-300. doi:10.1002/eat.20610

32. Sabel AL, Gaudiani JL, Statland B, Mehler PS. Hematological abnormalities in severe anorexia nervosa. Ann Hematol 2013; 92(5):605-613. doi:10.1007/s00277-013-1672-x

33. Boyar RM, Katz J, Finkelstein JW, et al. Anorexia nervosa. Immaturity of the 24-hour luteinizing hormone secretory pattern. N Engl J Med 1974; 291(17):861-865. doi:10.1056/NEJM197410242911701

34. Kimmel MC, Ferguson EH, Zerwas S, Bulik CM, Meltzer-Brody S. Obstetric and gynecologic problems associated with eating disorders. Int J Eat Disord 2016; 49(3):260-275. doi:10.1002/eat.22483

35. Mehler PS, Eckel RH, Donahoo WT. Leptin levels in restricting and purging anorectics. Int J Eat Disord 1999; 26(2):189-194. doi:10.1002/(sici)1098-108x(199909)26:2<189::aid-eat8>3.0.co;2-v

36. Rich LM, Caine MR, Findling JW, Shaker JL. Hypoglycemic coma in anorexia nervosa. Arch Intern Med 1990; 150(4):894-895. pmid:2183736

37. Drakin A, Rothman MS, Wassenaar E, Mascolo M, Mehler PS. Assessment and clinical management of bone disease in adults with eating disorders: a review. J Eat Disord 2017; 5:42. doi:10.1186/s40337-017-0172-0

38. Misra M, Golden NH, Katzman DK. State of the art systematic review of bone disease in anorexia nervosa. Int J Eat Disord 2016; 49(3):276-292. doi:10.1002/eat.22451

39. Fazeli PK, Klibanski A. Anorexia nervosa and bone metabolism. Bone 2014; 66:39-45. doi:10.1016/j.bone.2014.05.014

40. Lazaro L, Andres A, Calvo A, et al. Normal gray and white matter volume after weight restoration in adolescents with anorexia nervosa. Int J Eat Disord 2013; 46(8):841-848. doi:10.1002/eat.22161

41. Strumia R. Eating disorders and the skin. Clin Dermatol 2013; 31(1):80-85. doi:10.1016/j.clindermatol.2011.11.011

Address: Philip Mehler, MD, FACP, FAED, Denver Health Medical Center, 723 Delaware Street, Pav M, Denver, CO 80204; pmehler@dhha.org 